\title{
A new species of Lobellina and first record of Vietnura from China (Collembola: Neanuridae: Neanurinae)
}

\author{
Ji-Gang Jiang', Cheng-Wang Huang², Yun-Xia Luan³ \\ I College of Life and Environmental Sciences, Hunan University of Arts and Science, China 2 Institute of \\ Plant Physiology \& Ecology, Shanghai Institutes for Biological Sciences, Chinese Academy of Sciences, Shanghai \\ 200032, China 3 Guangzhou Key Laboratory of Insect Development Regulation and Application Research, In- \\ stitute of Insect Science and Technology \& School of Life Science, South China Normal University, Guangzhou \\ 510631, China \\ Corresponding author: Yun-Xia Luan (yxluan@scnu.edu.cn)
}

Academic editor:LouisDeharveng | Received 11 March 2018|Accepted30September2018| Published 17 December 2018

http://zoobank.org/B32CB55A-BBAB-424B-B1FA-245752E0478E

Citation: Jiang J-G, Huang C-W, Luan Y-X (2018) A new species of Lobellina and first record of Vietnura from China (Collembola: Neanuridae: Neanurinae). ZooKeys 807: 13-28. https://doi.org/10.3897/zookeys.807.24941

\begin{abstract}
A new species of Lobellina Yosii, 1956 and a key to all species of the genus is provided. It is distinguished from all known members of the genus by its unique set of morphological characters: mandible with six teeth, cephalic chaeta O present, and free from tubercle Fr, cephalic tubercle Oc with three chaetae, cephalic tubercle Di separate, and tubercle Dl with four (sometimes three) chaetae, Ant. I with eight chaetae, and claw with an inner tooth. Vietnura caerulea Deharveng \& Bedos, 2000 is recorded from China for the first time. New localities of Rambutanura hunanensis Jiang \& Dong, 2018 and Vitronura paraacuta Wang, Wang \& Jiang, 2016 from southwest China are also provided.
\end{abstract}

\section{Keywords}

key, Lobellina yinae sp. n., new records, taxonomy

\section{Introduction}

Maolan National Nature Reserve is located at Libo County, Qiannan Buyi and Miao Nationalities Autonomous Region of Guizhou Province, southwest China. It covers area of $212.85 \mathrm{~km}^{2}$ and is located in the subtropical monsoon humid climate zone. The main objectives of Maolan National Nature Reserve are the protection of the karst

Copyright Ji-Gang Jiang et al. This is an open access article distributed under the terms of the Creative Commons Attribution License (CC BY 4.0), which permits unrestricted use, distribution, and reproduction in any medium, provided the original author and source are credited. 
forest, and its rare animals and plants. It is from 430 to $1078 \mathrm{~m}$ above sea level. So far, no Neanuridae was reported from this reserve. During the field research at Maolan National Nature Reserve in 2015, four species of the subfamily Neanurinae were collected. They are described in the present paper.

\section{Materials and methods}

Specimens were extracted from soil samples with the aid of Tullgren funnels or directly collected with an aspirator, and preserved in 95\% ethanol. They were cleared in Nesbitt's fluid and mounted on slides in Hoyer's medium. Preparations were dried for 7-15 days in oven at $55^{\circ} \mathrm{C}$, then ringed with lacquer. The morphological characters were observed and figures were drawn using a phase contrast microscope Nikon 80i. Material is deposited in Shanghai Entomological Museum, Chinese Academy of Sciences.

The terminology and layout of the tables used in this paper follow Deharveng (1983), Deharveng and Weiner (1984), Smolis and Deharveng (2006), and Smolis (2008). The abbreviations used are listed below.

\section{General morphology}

$\begin{array}{llll}\text { Abd. } & \text { abdomen } & \mathrm{Scx} 2 & \text { subcoxa 2 } \\ \text { Ant. } & \text { antenna } & \mathrm{Ti} & \text { tibiotarsus } \\ \text { AOIII } & \text { sensory organ of antennal seg- } & \mathrm{Th} . & \text { thorax } \\ & \text { ment III } & \mathrm{Tr} & \text { trochanter } \\ \mathrm{Cx} & \text { coxa } & \mathrm{VT} & \text { ventral tube } \\ \mathrm{Fe} & \text { Femur } & & \end{array}$

\section{Groups of chaetae}

$\mathrm{Ag}$

An

ap

$\mathrm{ca}$

$\mathrm{cm}$

$\mathrm{cp}$

d

$\mathrm{Fu}$

Vc

antegenital
anal lobes
apical
centroapical
centromedial
centroposterior
dorsal
furcal
ventrocentral

Ve or ve

Vea

Vem

Vep

Vel

$\mathrm{Vec}$

Vei

Vi or vi

$\mathrm{Vl}$

$$
\begin{aligned}
& \text { ventroexternal } \\
& \text { ventroexternoanterior } \\
& \text { ventroexternomedial } \\
& \text { ventroexteroposterior } \\
& \text { ventroexternolateral } \\
& \text { ventroexternocentral } \\
& \text { ventroexternointernal } \\
& \text { ventrointernal } \\
& \text { ventrolateral }
\end{aligned}
$$

\section{Tubercles}

$\begin{array}{llll}\text { An } & \text { antennal } & \text { Di } & \text { dorsointernal } \\ \text { Fr } & \text { frontal } & \text { Dl } & \text { dorsolateral } \\ \text { Af } & \text { antenno-frontal } & \text { L } & \text { ateral } \\ \text { Cl } & \text { clypeal } & \text { Oc } & \text { ocular } \\ \text { De } & \text { dorsoexternal } & \text { So } & \text { subocular }\end{array}$




\section{Types of chaetae}

\begin{tabular}{|c|c|c|c|}
\hline $\mathrm{Ml}$ & long macrochaeta & or & organite of Ant. IV \\
\hline Mc & short macrochaeta & brs & border s-chaeta on Ant. IV \\
\hline Mcc & very short macrochaeta & 1 & ordinary chaeta on Ant. IV \\
\hline me & mesochaeta & mou & thin cylindrical chaetae on Ant. \\
\hline mi & microchaeta & & IV ("soies mousses") \\
\hline $\mathrm{ns}$ & s-microchaeta & $\mathrm{x}$ & labial sensory papilla \\
\hline & s-chaeta & & \\
\hline bs & s-chaeta on Ant. IV & $\mathrm{L}^{\prime}$ & ordinary lateral chaeta on Abd. \\
\hline miA & $\begin{array}{l}\text { microchaetae on Ant. IV } \\
\text { ordinary chaetae on ventral Ant. } \\
\text { IV }\end{array}$ & & 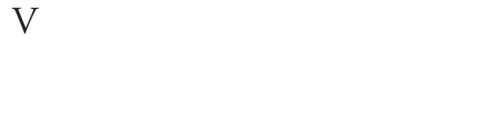 \\
\hline
\end{tabular}

\section{Taxonomy}

Tribe Lobellini Cassagnau, 1983

Genus Lobellina Yosii, 1956

\section{Lobellina yinae sp. $\mathrm{n}$.}

http://zoobank.org/FD11B2EC-7C4A-4480-BB09-BA7A3DEF144B

Figs 1-9, Tables 1-4

Material. Holotype, male, on slide. Maolan National Nature Reserve, Libo County, Guizhou Province, China. $25^{\circ} 16.400^{\prime} \mathrm{N}, 107^{\circ} 53.864^{\prime} \mathrm{E}$, ca. $780 \mathrm{~m}$ above sea level, 22 July 2015. Collected by Cheng-Wang Huang, Yan Liang and Ai-Min Liu. Paratype, one subadult, same slide and data as holotype.

Etymology. The species is named after Prof. Wen-Ying Yin, in honor of her important contributions to the study of Chinese soil animals.

Diagnosis. Three pigmented eyes, mandible with six teeth, cephalic chaeta $\mathrm{O}$ present and free from tubercle Fr, cephalic tubercle Oc with three chaetae, cephalic tubercle Di separate, tubercle Dl with four (sometimes three) chaetae, Ant. I with eight chaetae, and claw with single inner tooth.

Description. General (Figs 1-3). Body length (without antenna) $1.8-2.1 \mathrm{~mm}$. Cuticular granulations medium, tertiary granules absent, body without reticulations. Tubercles well developed on dorsal side of body. Body color red when alive, white in alcohol. Eyes 3+3, pigmented (Fig. 1). Chaetal morphology (Fig. 9). Dorsal ordinary chaetae of five types: Ml, Mc, Mcc, me, and mi. Macrochaetae Ml long, sheathed, weakly toothed and knobbed at apex. Macrochaetae Mc morphologically of two types: one is similar to Ml, but shorter, the other one with slightly pointed apex. Macrochaetae Mcc morphologically similar to $\mathrm{Ml}$ and shorter than Mc. Mesochaetae similar to ventral chaetae, thin, smooth, and pointed, with various length. Microchaetae shorter than mesochaetae, with acuminate tip. S-chaetae on terga thin, smooth, shorter than Mc, longer than Mcc. Antenna (Fig. 4 and Table 3). Antenna 4-segmented. Ant. I with eight 


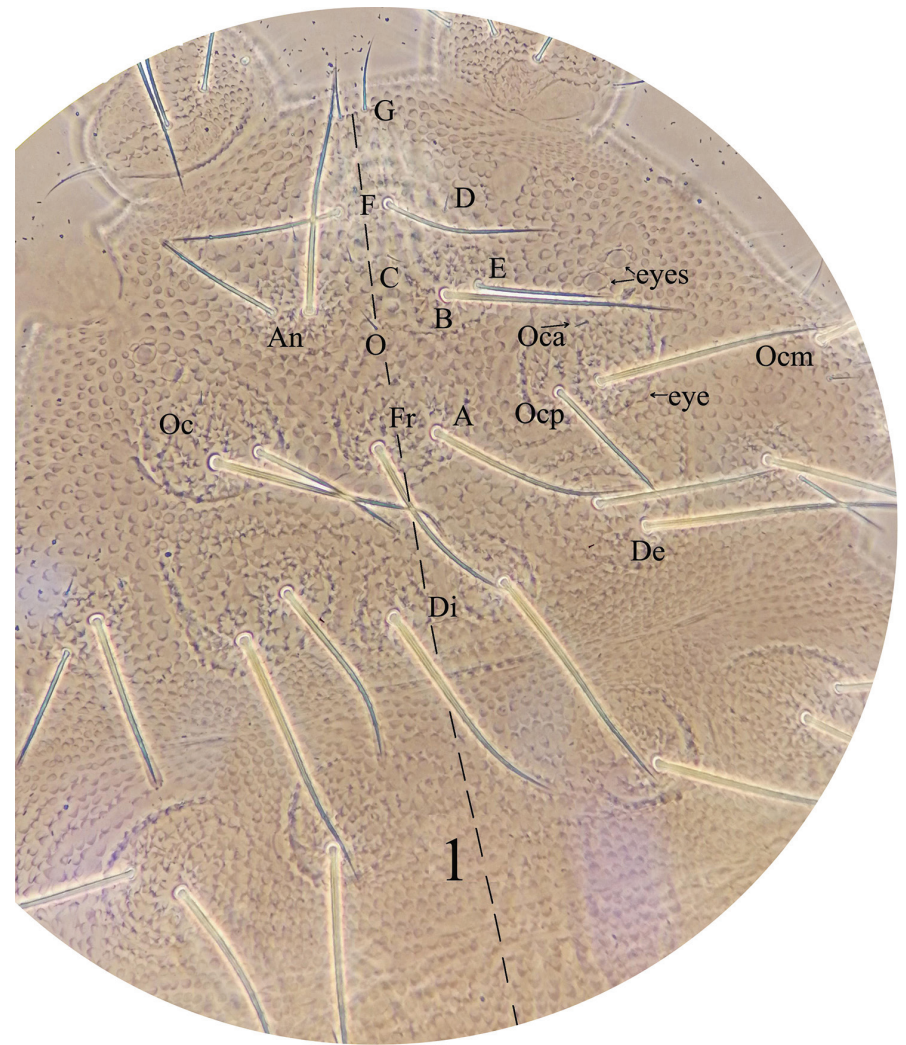

Figure I. Lobellina yinae sp. n. dorsal tubercle and chaetotaxy of head.

chaetae. Ant. II with eleven chaetae and dorsally with a smooth circular area. Ant. III dorsally fused to Ant. IV. AOIII consists of two short rods, ventral ms and two longer sensory chaetae (sgd and sgv), sgd on the same level position of the two rods, each rod exposed in separate pit. Ant. IV dorsally with eight thickened and blunt sensilla, slender i-chaeta, and minute capitate organite (or). Apical bulb distinct, trilobed. Each of the eight sensilla distinctly differentiated, larger and two times shorter than "mou"-chaetae. Ventral chaetotaxy of Ant. III-IV is shown in Table 3, ap with eight bs and three miA, ca with two bs and two miA, cm with three bs and one miA, cp with six bs and seven miA. On ventral side of Ant. III, Vi, Vc, Ve respectively with four, four, five chaetae, Ant. III dorsally with $4-5 \mathrm{~d}$ chaetae, $\mathrm{d} 1, \mathrm{~d} 2$, $\mathrm{d} 3$ as me, $\mathrm{d} 4 \mathrm{as}$ mi, $\mathrm{d} 5$ as mi and sometimes absent. Mouthparts. Buccal cone moderately long, labrum ventral sclerifications truncated (Fig. 8). Labrum chaetotaxy: 0/2, 2. Labium with normal chaetotaxy, and chaeta $\mathrm{F}$ almost three times as long as chaeta $\mathrm{A}$, without papillae $\mathrm{x}$ (Fig. 8). Maxilla styliform, consisting of two fused lamellae, apically with two tiny teeth (Fig. 7). Mandible with four apical teeth, one middle tooth, and one large basal tooth (Fig. 6). Dorsal chaetotaxy and tubercles of head (Fig. 1 and Table 1). Head with 14 tubercles. Tubercle $\mathrm{Cl}$ with four chaetae: 2G+2F; tubercle An with four chaetae: B, C, D, E; tubercle Oc with three chaetae; tubercle Fr with three chaetae, chaeta $\mathrm{O}$ present, shifting between the two tubercles 


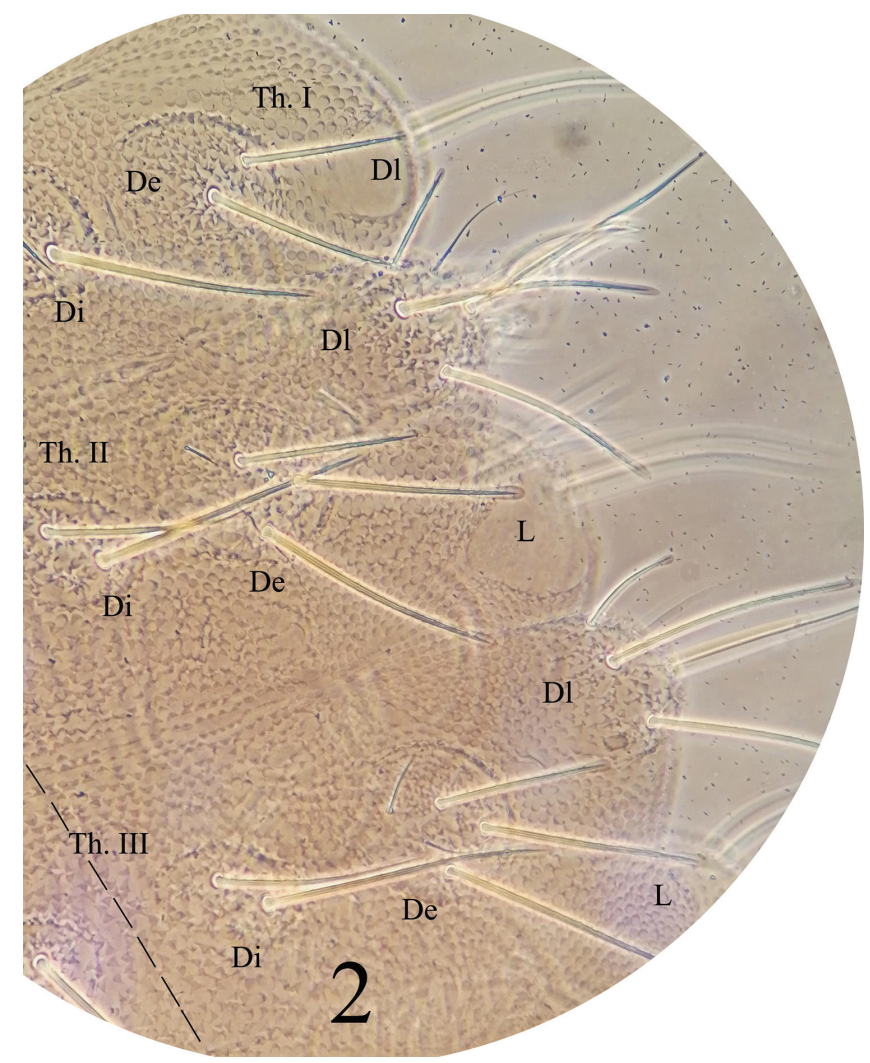

Figure 2. Lobellina yinae sp. n. dorsal tubercles and chaetotaxy on Th. I-III.

An; tubercle Di with a single chaeta; De with three chaetae; tubercle Dl separate from tubercle L+So, with four (or three) chaetae; tubercle L+So with 13 chaetae. Dorsal chaetotaxy and tubercles of thorax (Fig. 2 and Table 4). Thoracic dorsal tubercles complete. Th. I with three tubercles, tubercle Di with one chaeta; tubercle De with two chaetae; tubercle Dl with one chaeta. Th. II with four tubercles, tubercle Di with three chaetae; tubercle De with five chaetae $(4+s)$; tubercle Dl with five chaetae and one ms $(4+s+m s)$; tubercle L with three chaetae. Th. III with four tubercles, tubercle Di with three chaetae; tubercle De with five chaetae (4+s); tubercle Dl with five chaetae $(4+s)$; tubercle L with three chaetae. Dorsal chaetotaxy and tubercles of abdomen (Fig. 3 and Table 4). Dorsum of Abd. I with four tubercles, tubercle Di with two chaetae; tubercle De with four chaetae $(3+s)$; tubercle Dl with three chaetae; tubercle L with four chaetae. Tubercles and chaetae arrangements of Abd. II-III as on Abd. I. Abd. IV with four tubercles, tubercle Di with two chaetae; tubercle De with three chaetae $(2+s)$; tubercle Dl with three chaetae; tubercle L with 5-7 chaetae. Abd. V with four tubercles, tubercle Di with three chaetae; tubercle De with one chaeta $(s)$; tubercle $\mathrm{Dl}$ with four chaetae; tubercle $\mathrm{L}$ with seven chaetae (without s chaeta). Abd. VI bilobed, each side of Abd. VI with one tubercle, each tubercle with seven chaetae. No cryptopygy. S-chaetae formula on tergites as 0 , 2+ms, 2/1, 1, 1, 1, 1. Ventral chaetotaxy (Fig. 5, Table 2). On ventral side of head, groups 
Table I. Cephalic dorsal tubercles and chaetotaxy of Lobellina yinae sp. n.

\begin{tabular}{|c|c|c|c|}
\hline Tubercle & Number of chaetae & Types of chaetae & Names of chaetae \\
\hline \multirow{2}{*}{$\mathrm{Cl}$} & \multirow{2}{*}{4} & Ml & F \\
\hline & & me & G \\
\hline \multirow{3}{*}{ An } & \multirow{3}{*}{4} & $\mathrm{M}$ & B \\
\hline & & Mcc & E \\
\hline & & me & C, D \\
\hline \multirow{2}{*}{$\mathrm{Fr}$} & \multirow{2}{*}{3} & $\mathrm{Ml}$ & A \\
\hline & & me & $\mathrm{O}$ \\
\hline \multirow{3}{*}{ Oc } & \multirow{3}{*}{3} & Ml & $\mathrm{Ocm}$ \\
\hline & & Mcc & Ocp \\
\hline & & me or mi & Oca \\
\hline \multirow{2}{*}{ Di } & \multirow{2}{*}{1} & \multirow{2}{*}{ Ml } & Di1 \\
\hline & & & Chaetal homology uncertain \\
\hline \multirow{3}{*}{ De } & \multirow{3}{*}{3} & $\mathrm{Ml}$ & De1 \\
\hline & & Mc & De2 \\
\hline & & $\mathrm{mi}$ & Di2 \\
\hline $\mathrm{Dl}$ & $4(3)$ & $\mathrm{Mc}+\mathrm{Mcc}+2 \mathrm{me}($ or $\mathrm{mi})$ & Chaetal homology uncertain \\
\hline L+So & 13 & $4 \mathrm{Ml}+9 \mathrm{me}$ & Chaetal homology uncertain \\
\hline
\end{tabular}

Table 2. Cephalic ventral chaetotaxy of Lobellina yinae sp. n.

\begin{tabular}{l|c}
\hline \multicolumn{1}{c|}{ Group } & Number of chaetae \\
\hline Vi & 5 \\
\hline Vea & 5 \\
\hline Vem & 4 \\
\hline Vep & 4 \\
\hline Labium & $11,0 \mathrm{X}$ \\
\hline
\end{tabular}

Table 3. Chaetotaxy of antenna of Lobellina yinae sp. $\mathrm{n}$.

\begin{tabular}{|c|c|c|c|}
\hline Segment, group & Number of chaetae & Segment, group & Number of chaetae \\
\hline I & 8 & \multirow{3}{*}{ IV } & \multirow{3}{*}{ or, $8 \mathrm{~s}, 12 \mathrm{mou}$, ? brs, 2 iv } \\
\hline II & 11 & & \\
\hline III & 5 sensilla AOIII & & \\
\hline $\mathrm{Ve}$ & 5 & ap & $8 \mathrm{bs}, 3 \mathrm{miA}$ \\
\hline $\mathrm{Vc}_{\mathrm{c}}$ & 4 & $\mathrm{ca}$ & $2 \mathrm{bs}, 2 \mathrm{miA}$ \\
\hline $\mathrm{Vi}$ & 4 & $\mathrm{~cm}$ & $3 \mathrm{bs}, 1 \mathrm{miA}$ \\
\hline $\mathrm{d}$ & $4(2 m e+2 m i)-5(2 m e+3 m i)$ & $\mathrm{cp}$ & $1 \mathrm{brs}, 7 \mathrm{miA}$ \\
\hline
\end{tabular}

Vea, Vem, and Vep with five, four, four chaetae respectively. Group Vi on head with five chaetae. On Abd. I, VT with one proximal and three distal chaetae. On Abd. III, furca rudimentary with three chaetae, and without microchaeta. On Abd IV, group Vei, Vec, Vel respectively with one, two, four chaetae. On Abd. V, group Vl with 2-3 chaetae, Ag with 3-4 chaetae, chaeta L' absent. Anal lobe with 14-15 chaetae and three mi. Legs (Table 4). Unguis with an inner tooth and without lateral tooth. Chaeta $M$ on tibiotarsus present. Tibiotarsus of foreleg, midleg, and hindleg with 19, 19, 18 chaetae respectively.

Ecology and distribution. In fallen leaves of bamboo. Lobellina yinae sp. $n$. is only known from Libo (Fig. 16). 
Table 4. Postcephalic tubercles and chaetotaxy of Lobellina yinae sp. n.

\begin{tabular}{|c|c|c|c|c|c|c|c|c|c|}
\hline \multicolumn{5}{|c|}{ Terga } & \multicolumn{5}{|c|}{ Legs } \\
\hline & Di & $\mathrm{De}$ & $\mathrm{Dl}$ & $\mathrm{L}$ & Scx2 & $\mathrm{Cx}$ & $\operatorname{Tr}$ & $\mathrm{Fe}$ & $\mathrm{T}$ \\
\hline Th. I & $\mathrm{Ml}$ & $\mathrm{Ml}+\mathrm{me}$ & Ml & - & 0 & 3 & 6 & 13 & 19 \\
\hline Th. II & $\mathrm{Ml}+\mathrm{Mc}+\mathrm{mi}$ & $\mathrm{Ml}+\mathrm{Mc}+\mathrm{Mcc}+\mathrm{me}+\mathrm{s}$ & $3 \mathrm{Ml}+\mathrm{Mcc}+\mathrm{s}+\mathrm{ms}$ & $\mathrm{Ml}+2 \mathrm{Mcc}$ & 2 & 7 & 6 & 12 & 19 \\
\hline Th. III & $\mathrm{Ml}+\mathrm{Mc}+\mathrm{mi}$ & $\mathrm{Ml}+\mathrm{Mc}+\mathrm{Mcc}+\mathrm{me}+\mathrm{s}$ & $3 \mathrm{Ml}+\mathrm{Mcc}+\mathrm{s}$ & $\mathrm{Ml}+2 \mathrm{Mcc}$ & 2 & 8 & 6 & 11 & 18 \\
\hline \multicolumn{5}{|c|}{ Terga } & \multicolumn{5}{|c|}{ Sterna } \\
\hline Abd. I & $\mathrm{Ml}+\mathrm{Mc}$ & $\mathrm{Ml}+\mathrm{Mc}+\mathrm{me}+\mathrm{s}$ & $\mathrm{Ml}+\mathrm{Mc}+\mathrm{Mcc}$ & $\mathrm{Ml}+\mathrm{Mc}+2 \mathrm{me}$ & \multicolumn{5}{|c|}{ VT: 4} \\
\hline Abd. II & $\mathrm{Ml}+\mathrm{Mc}$ & $\mathrm{Ml}+\mathrm{Mc}+\mathrm{me}+\mathrm{s}$ & $\mathrm{Ml}+\mathrm{Mc}+\mathrm{Mcc}$ & $\mathrm{Ml}+\mathrm{Mc}+2 \mathrm{me}$ & \multicolumn{5}{|c|}{ Ve: 4-5, V1: 0} \\
\hline Abd. III & $\mathrm{Ml}+\mathrm{Mc}$ & $\mathrm{Ml}+\mathrm{Mc}+\mathrm{me}+\mathrm{s}$ & $\mathrm{Ml}+\mathrm{Mc}+\mathrm{Mcc}$ & $\mathrm{Ml}+\mathrm{Mc}+3 \mathrm{me}$ & \multicolumn{5}{|c|}{ Ve: 4, Fu: 3, $0 \mathrm{mi}$} \\
\hline Abd. IV & $\mathrm{Ml}+\mathrm{Mc}$ & $\mathrm{Ml}+\mathrm{Mc}+\mathrm{s}$ & $\mathrm{Ml}+\mathrm{Mc}+\mathrm{Mcc}$ & $\begin{array}{c}3 \mathrm{Ml}+2 \mathrm{me} \text { or } \\
(3 \mathrm{Ml}+2 \mathrm{me}+2 \mathrm{Mc})\end{array}$ & \multicolumn{5}{|c|}{ Vei: 1, Vec: 1, Vel: 2 , Vl: 4} \\
\hline Abd. V & $\mathrm{Ml}+\mathrm{Mc}+\mathrm{me}$ & $s$ & $2 \mathrm{Ml}+\mathrm{Mc}+\mathrm{Mcc}$ & $7 \mathrm{me}$ or $1 \mathrm{Mc}+6 \mathrm{me}$ & \multicolumn{5}{|c|}{ Ag:3-4, Vl: 2-3, L': 0} \\
\hline Abd. VI & \multicolumn{4}{|c|}{$2 \mathrm{Ml}+5 \mathrm{me}$} & \multicolumn{5}{|c|}{ Ve: $14-15$, An: $3 \mathrm{mi}$} \\
\hline
\end{tabular}

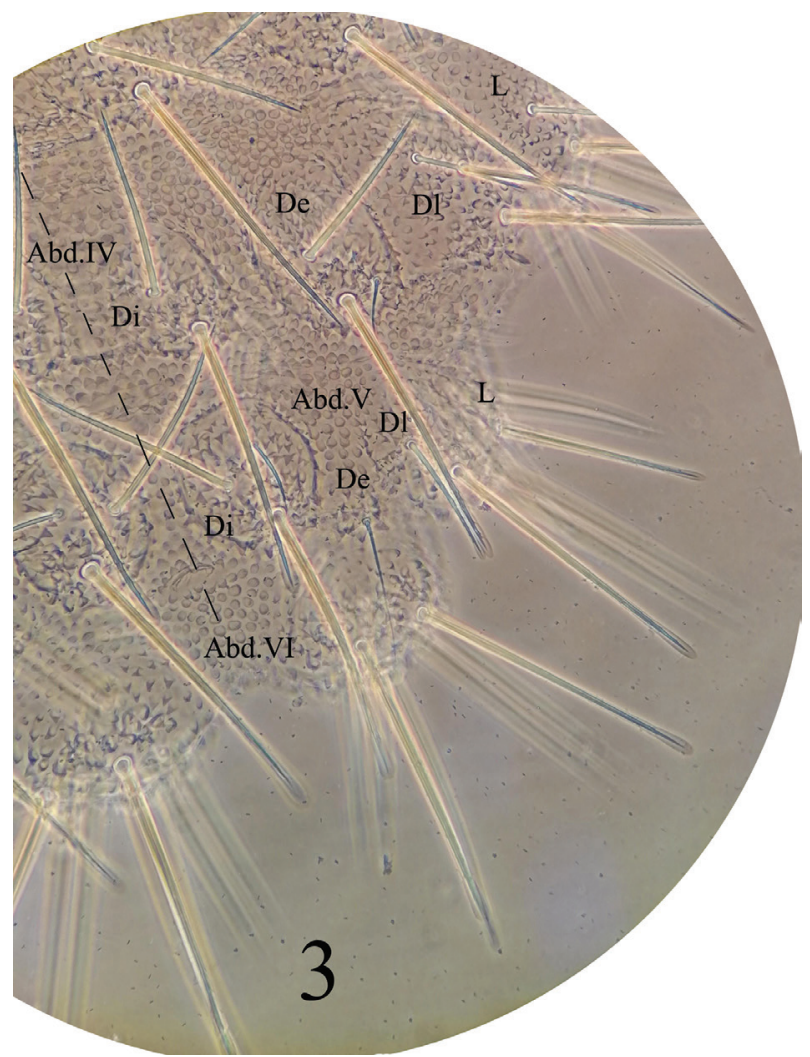

Figure 3. Lobellina yinae sp. n. dorsal tubercles and chaetotaxy on Abd. IV-VI.

Remarks. To date, 15 species of the genus Lobellina are known from Asia and one from Central America (Cuba) (Deharveng and Weiner 1984, Ma and Chen 2008, Smolis 2017, Jiang et al. 2018). The new species is similar to L. montana Deharveng 


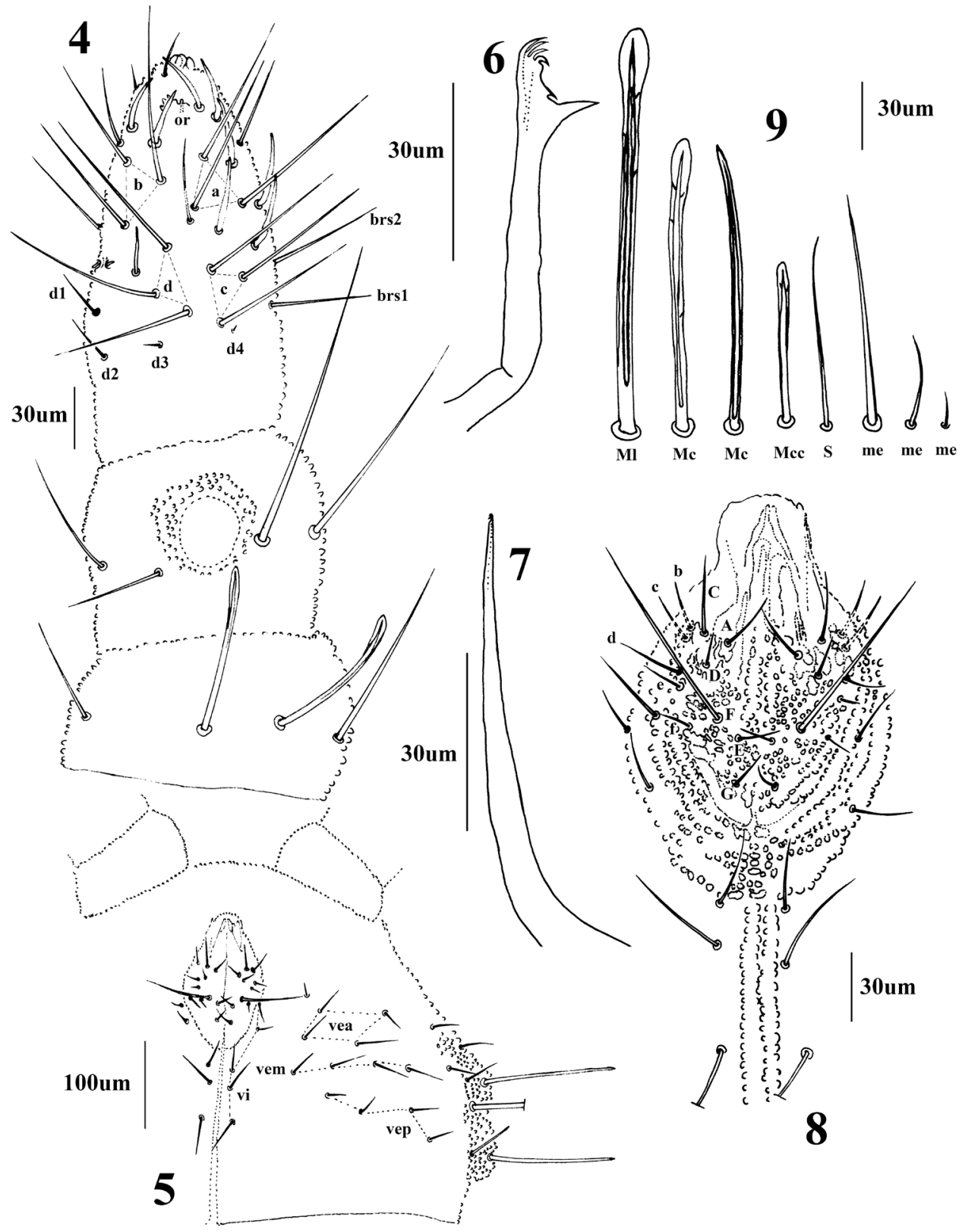

Figures 4-9. Lobellina yinae sp. n. 4 dorsal chaetotaxy of antenna $\mathbf{5}$ ventral chaetotaxy of head $\mathbf{6}$ mandible 7 maxilla 8 Labium 9 types of body chaetae.

\& Weiner, 1984 and L. paraminuta Deharveng \& Weiner, 1984 from Korea by the following characters: cephalic chaeta $\mathrm{O}$ free from tubercle Fr (shifting between two tubercles An), cephalic tubercle Dl separate from tubercle $\mathrm{L}+\mathrm{So}$, tubercle Oc with three 
chaetae, Abd. V with 3+3 dorsal tubercles and De separate from $\mathrm{Dl}$, and claw with a distinct basal inner tooth. However, L. yinae sp. n. can be distinguished from L. montana and $L$. paraminuta by its mandible with six teeth versus seven, cephalic tubercle $\mathrm{Dl}$ with three or four chaetae versus five, tubercle De on Abd. I-III with four chaetae $(3+s)$ versus three $(2+s)$, and tubercle $\mathrm{Dl}$ on Abd. I-III with three chaetae versus two.

The new species is also similar to L. fusa Jiang, Wang \& Xia, 2018 from China by the following characters: mandible with six teeth, maxilla styliform, tubercle $\mathrm{Fr}$ on head with three chaetae, tubercle Oc on head with three chaetae, Abd.V with 3+3 dorsal tubercles and De separate from $\mathrm{Dl}$, and claw with a distinct basal inner tooth. However, the new species can be differentiated from L. fusa by the cephalic chaeta $\mathrm{O}$ of tubercle Fr free (not free in L. fusa), cephalic tubercles Di separated (fused in L. fusa), cephalic tubercle $\mathrm{Dl}$ with four chaetae (five in $L$. fusa), and each tubercle $\mathrm{Dl}$ on Abd. I-III with three chaetae (two chaetae in L. fusa).

Key to species of the genus Lobellina Yosii, 1956 (Modified from Jiang et al. 2018)

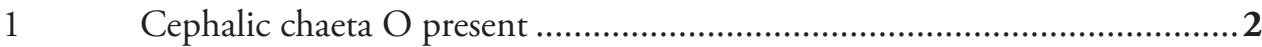

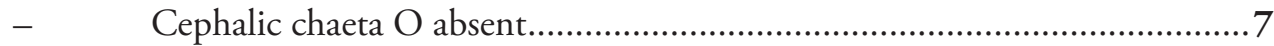

$2 \quad$ Chaeta $\mathrm{O}$ included in tubercle Fr............................................................. 3

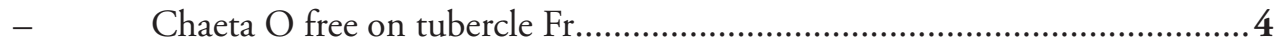

3 Bodycoloryellow, mandible with seven teeth, tubercle Ocwith 2 chaetae, ventral tube with $5+5$ chaetae, cephalic tubercles Di separate

L. nanjingensis Ma \& Chen, 2008 (China)

- $\quad$ Body color red, mandible with six teeth, tubercle Oc with three chaetae, ventral tube with $4+4$ chaetae, cephalic tubercles Di fused

L. fusa Jiang, Wang \& Xia, 2018 (China)

Mandible with six teeth, Cephalic tubercle Dl with four (or three) chaetae... L. yinae sp. n. (China)

Mandible with seven teeth, Cephalic tubercle $\mathrm{Dl}$ with five chaetae . .5 Tubercle $\mathrm{Dl}$ on $\mathrm{Th}$. II with six chaetae $(4+s+\mathrm{ms})$

L. montana Deharveng \& Weiner, 1984 (Korea)

Tubercle $\mathrm{Dl}$ on Th. II with five chaetae $(3+\mathrm{s}+\mathrm{ms})$

Body macrochaetae smooth

- $\quad$ Body macrochaetae serrate...... 
9 Abd. V with 2+2 dorsal tubercles

- $\quad$ Abd. V with 3+3 dorsal tubercles

L. chosonica Deharveng \& Weiner, 1984 (Korea)

L. proxima Deharveng \& Weiner, 1984 (Korea)

10 Tubercle Di on Abd. V with two chaetae ..................................................11

- $\quad$ Tubercle Di on Abd. V with three chaetae ....... L. minuta (Lee, 1980) (Korea)

11 Mandible with three teeth.................. L. ipohensis (Yosii, 1976) (Malaysia)

- $\quad$ Mandible with 6-8 teeth ..................................................................12

12 Mandible with six teeth, tubercle De+Dl with six chaetae $(5+s)$

L. pomorskii Smolis, 2017 (Vietnam)

- $\quad$ Mandible with eight teeth, tubercle De+Dl with five chaetae $(4+s)$

13 Cephalic tubercle Oc with two chaetae .................................................14

- Cephalic tubercle Oc with three chaetae ................................................15

14 Abd. V with 2+2 dorsal tubercles

L. ionescui (Massoud \& Gruia, 1974) (Cuba)

Abd. $V$ with $3+3$ dorsal tubercles

L. perfusionides (Stach, 1965) (Vietnam)

Abd. $V$ with $2+2$ dorsal tubercles ............... L. roseola (Yosii, 1954) (Japan)

Abd. V with 3+3 dorsal tubercles .......... L. kitazawai (Yosii, 1969) (Japan)

Tribe Neanurini Börner, 1901 (sensu Cassagnau, 1983)

Genus Vietnura Deharveng \& Bedos, 2000: new record to China

Vietnura caerulea Deharveng \& Bedos, 2000: 209-214, figs 1-4 (Vietnam) new record to China

Material. Two males on the same slide, one of them submature, $25^{\circ} 17.453^{\prime} \mathrm{N}$, $107^{\circ} 56.359^{\prime} \mathrm{E}$, elevation $880-900 \mathrm{~m}$. Three individuals in alcohol, Coordinates: $25^{\circ} 17.516^{\prime} \mathrm{N}, 107^{\circ} 56.371^{\prime} \mathrm{E}$, elevation $840 \mathrm{~m}$. One specimen in alcohol, $25^{\circ} 17.483^{\prime} \mathrm{N}$, $107^{\circ} 56.245^{\prime} \mathrm{E}$, elevation $731 \mathrm{~m}$. All of them were collected by Cheng-Wang Huang, Yan Liang \& Ai-Min Liu, from Maolan National Nature Reserve, Libo County, Guizhou Province, China, on 19 July 2015. Material deposited in Shanghai Entomological Museum, Chinese Academy of Sciences.

Description of the Chinese specimens (Figs 10-15, Tables 5-7). Body length (without antenna) 0.9-1.1 mm. Cuticular granulations medium, tertiary granules developed, body with reticulations. Tubercles well developed on dorsal side of body. Body color blue alive and in alcohol. Eyes $2+2$, small and pigmented, all on tubercles Oc. Chaetal morphology (Fig. 14). Dorsal ordinary chaetae of four types: Ml, Mc, Mcc, and me. Macrochaetae Ml long, sheathed, distinctly toothed and knobbed at apex (Fig. 14a). Macrochaetae Mc morphologically similar to long macrochae- 
Table 5. Cephalic ventral chaetotaxy of Vietnura caerulea Deharveng \& Bedos, 2000.

\begin{tabular}{l|c}
\hline \multicolumn{1}{c|}{ Group } & Number of chaetae \\
\hline Vi & 5 \\
\hline Vea & 2 \\
\hline Vem & 2 \\
\hline Vep & 2 \\
\hline Labium & $11,0 \times$ \\
\hline
\end{tabular}

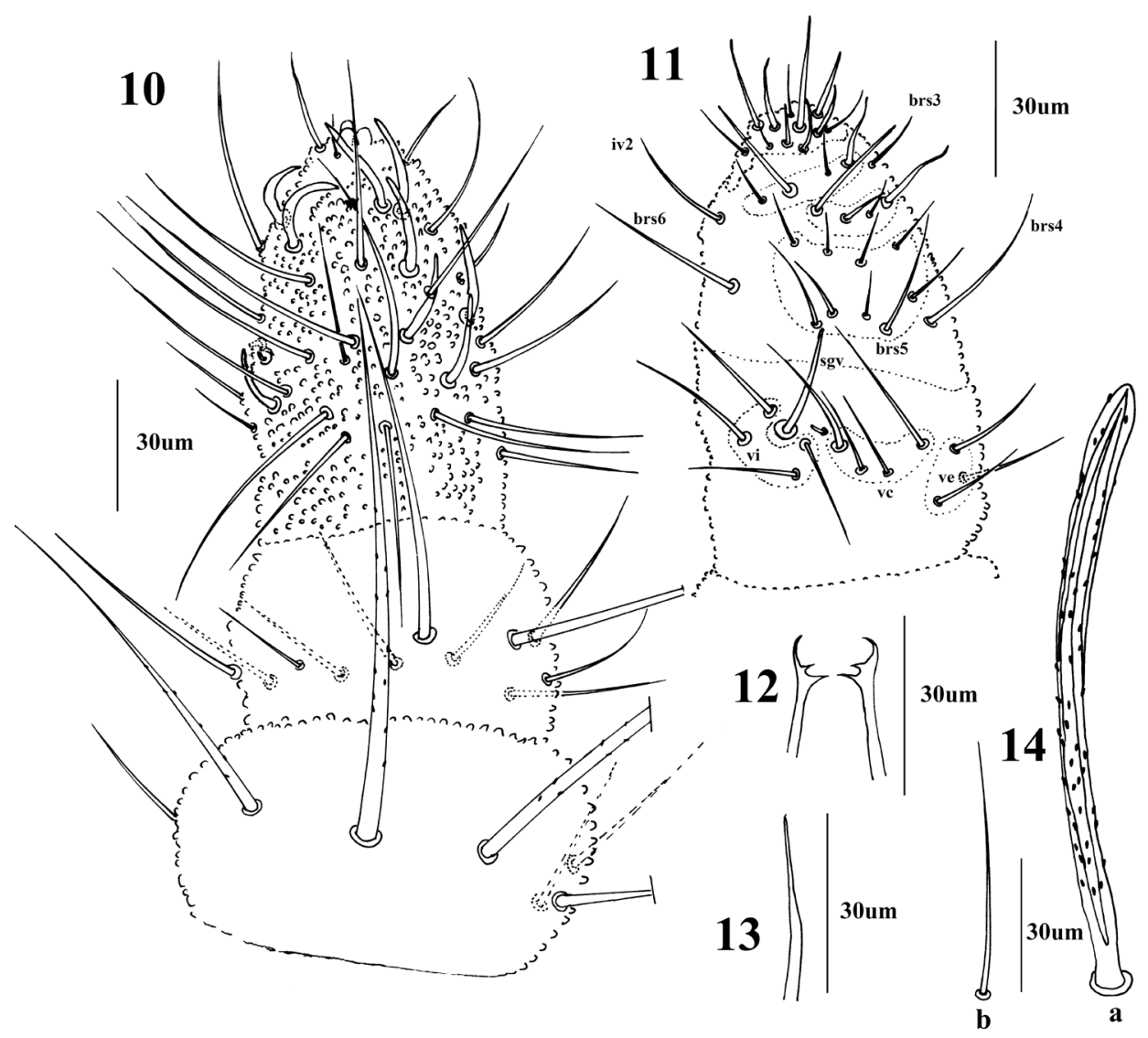

Figures 10-14. Vietnura caerulea Deharveng \& Bedos, 2000 I0 dorsal side of antenna I I ventral side of Ant. III-IV I 2 mandible $\mathbf{3}$ maxilla $\mathbf{4}$ body setae, a: macrochaeta, b: S-chaeta.

tae, but shorter. Macrochaetae Mcc morphologically similar to Mc and shorter than Mc. Mesochaetae similar to ventral chaetae, thin, smooth, and pointed, with various lengths. S-chaetae of tergites thin, smooth, shorter than Mc and slightly longer than Mcc "mou" (Fig. 14b). S-chaetae formula on tergites as 0, 2+ms, 2/1, 1, 1, 1, 1. Antenna. Antenna 4-segmented. Ant. I with seven chaetae. Ant. II with 10-11 chaetae. Ant. III dorsally fused to Ant. IV. AOIII consists of two short rods, one ventral ms 
Table 6. Chaetotaxy of antenna of Vietnura caerulea Deharveng \& Bedos, 2000.

\begin{tabular}{l|c|c|c}
\hline Segment, group & Number of chaetae & Segment, group & Number of chaetae \\
\cline { 1 - 2 } I & 7 & IV & or, $8 \mathrm{~s}, 12 \mathrm{mou}$, ? brs, 2 iv \\
\cline { 1 - 2 } II & $10-11$ & & $7 \mathrm{bs}, 4 \mathrm{miA}$ \\
\hline $\mathrm{III}$ & 3 & $\mathrm{ap}$ & $2 \mathrm{bs}, 2 \mathrm{miA}$ \\
\hline $\mathrm{Ve}$ & 4 & $\mathrm{ca}$ & $3 \mathrm{bs}, 1 \mathrm{miA}$ \\
\hline $\mathrm{Vc}$ & 4 & $\mathrm{~cm}$ & $1 \mathrm{brs}, 8 \mathrm{miA}$ \\
\hline $\mathrm{di}$ & 3 & $\mathrm{cp}$ & \\
\hline
\end{tabular}

Table 7. Postcephalic tubercles and chaetotaxy of Vietnura caerulea Deharveng \& Bedos, 2000.

\begin{tabular}{|c|c|c|c|c|c|c|c|c|c|}
\hline \multicolumn{5}{|c|}{ Terga } & \multicolumn{5}{|c|}{ Legs } \\
\hline & $\mathrm{Di}$ & De & $\mathrm{Dl}$ & $\mathrm{L}$ & Scx2 & $\mathrm{Cx}$ & $\mathrm{Tr}$ & $\mathrm{Fe}$ & $\mathrm{T}$ \\
\hline Th. I & Mc & $\mathrm{Mc}+\mathrm{Mcc}$ & Mc & - & 0 & 3 & 6 & 13 & 19 \\
\hline Th. II & $\mathrm{Ml}+\mathrm{Mcc}$ & $\mathrm{Mc}+\mathrm{Mcc}_{\mathrm{s}} \mathrm{s}$ & $\mathrm{Ml}+2 \mathrm{Mcc}+\mathrm{s}+\mathrm{ms}$ & $\mathrm{Ml}+\mathrm{Mc}+\mathrm{Mcc}$ & 2 & 7 & 6 & 12 & 19 \\
\hline Th. III & $\mathrm{Ml}+\mathrm{Mcc}$ or $\mathrm{Ml}+2 \mathrm{Mcc}$ & $\mathrm{Mc}+\mathrm{Mcc}+\mathrm{s}$ & $\mathrm{Ml}+2 \mathrm{Mcc}+\mathrm{s}$ & $\mathrm{Ml}+\mathrm{Mc}+\mathrm{Mcc}$ & 2 & 8 & 6 & 11 & 18 \\
\hline \multicolumn{5}{|c|}{ Terga } & \multicolumn{5}{|c|}{ Sterna } \\
\hline Abd. I & $\mathrm{Ml}+\mathrm{Mcc}$ & $\mathrm{Ml}+\mathrm{Mcc}+\mathrm{s}$ & $\mathrm{Ml}+\mathrm{Mcc}$ & $\mathrm{Ml}+\mathrm{Mcc}+\mathrm{me}$ & \multicolumn{5}{|c|}{ VT: 4} \\
\hline Abd. II & $\mathrm{Ml}+\mathrm{Mcc}$ & $\mathrm{Ml}+\mathrm{Mcc}+\mathrm{s}$ & $\mathrm{Ml}+\mathrm{Mcc}$ & $\mathrm{Ml}+\mathrm{Mcc}+\mathrm{me}$ & \multicolumn{5}{|c|}{ Ve: 3} \\
\hline Abd. III & $\mathrm{Ml}+\mathrm{Mcc}$ & $\mathrm{Ml}+\mathrm{Mcc}+\mathrm{s}$ & $\mathrm{Ml}+\mathrm{Mcc}$ & $\mathrm{Ml}+\mathrm{Mcc}+\mathrm{me}$ & \multirow{2}{*}{\multicolumn{5}{|c|}{\begin{tabular}{|l} 
Ve: $3-4$, Fu: $3-4$ me, mi: 0 \\
Vei: 1 , Vec: 2 , Vel: 3, Vl: $3-4$
\end{tabular}}} \\
\hline Abd. IV & $\mathrm{Ml}+\mathrm{Mcc}$ & $\mathrm{Ml}+\mathrm{Mcc}+\mathrm{s}$ & $\mathrm{Ml}+\mathrm{Mcc}$ & $4 \mathrm{me}$ & & & & & \\
\hline Abd. V & $2(\mathrm{Ml}+\mathrm{Mcc})^{*}$ & \multicolumn{3}{|c|}{$\mathrm{Ml}+\mathrm{Mcc}+2 \mathrm{me}+\mathrm{s}$} & \multicolumn{5}{|c|}{ Ag: $2, \mathrm{Vl}: 2$} \\
\hline Abd. VI & \multicolumn{4}{|c|}{$7(8)$} & \multicolumn{5}{|c|}{ Ve: 12, An: $1 \mathrm{mi}$} \\
\hline
\end{tabular}

*2 Di fused.

and two longer sensilla (sgd and sgv), sgd shifted basally to the back of the two rods, each rod exposed in separate pit (Fig. 10). Ant. IV dorsally with eight sensilla, slender i-chaeta, and minute capitate organite (or), apical bulb small, trilobed (Fig. 10). Sensilla thicker and shorter than "mou"-chaetae (Fig. 10). Ventral chaetotaxy of Ant. III-IV as in Fig. 11 and Table 6, group ap with seven bs and four miA, ca with two bs and two miA, cm with three bs and one miA, cp with eight miA and brs5. On ventral side of Ant. III, Vi, Vc, Ve respectively with four, four, three chaetae; dorsally with three d chaetae, d3 as mesochaeta (Fig. 10). Mouthparts. Buccal cone short, labral sclerifications not ogival. Labrum chaetotaxy: ?/2, 4. Labium with four basal, three distal, four lateral chaetae, without papillae x. Maxilla reduced, styliform (Fig. 13). Mandible reduced, tridentate (Fig. 12).

Dorsal chaetotaxy and tubercles (Table 7). Head with six tubercles. Tubercle $\mathrm{Cl}$ with four chaetae: two G and two F; tubercle Af+Oc with four chaetae: two B and two Ocm, chaeta $\mathrm{O}$ absent; tubercle $\mathrm{Di}+\mathrm{De}$ with four chaetae: two Di1, two De1; tubercle $\mathrm{Dl}+\mathrm{L}+$ So with eleven chaetae $(5 \mathrm{Ml}+6 \mathrm{me})$. Thorax and abdomen tubercles and chaetotaxy as in Table 7. Cryptopygy.

Ventral chaetotaxy (Fig. 15 and Table 5). On head, groups Vea, Vem and Vep with two, two, two chaetae respectively. Group Vi on head with five chaetae. VT with one proximal and three distal chaetae. On Abd. III, furca rudimentary with 3-4 chaetae, 


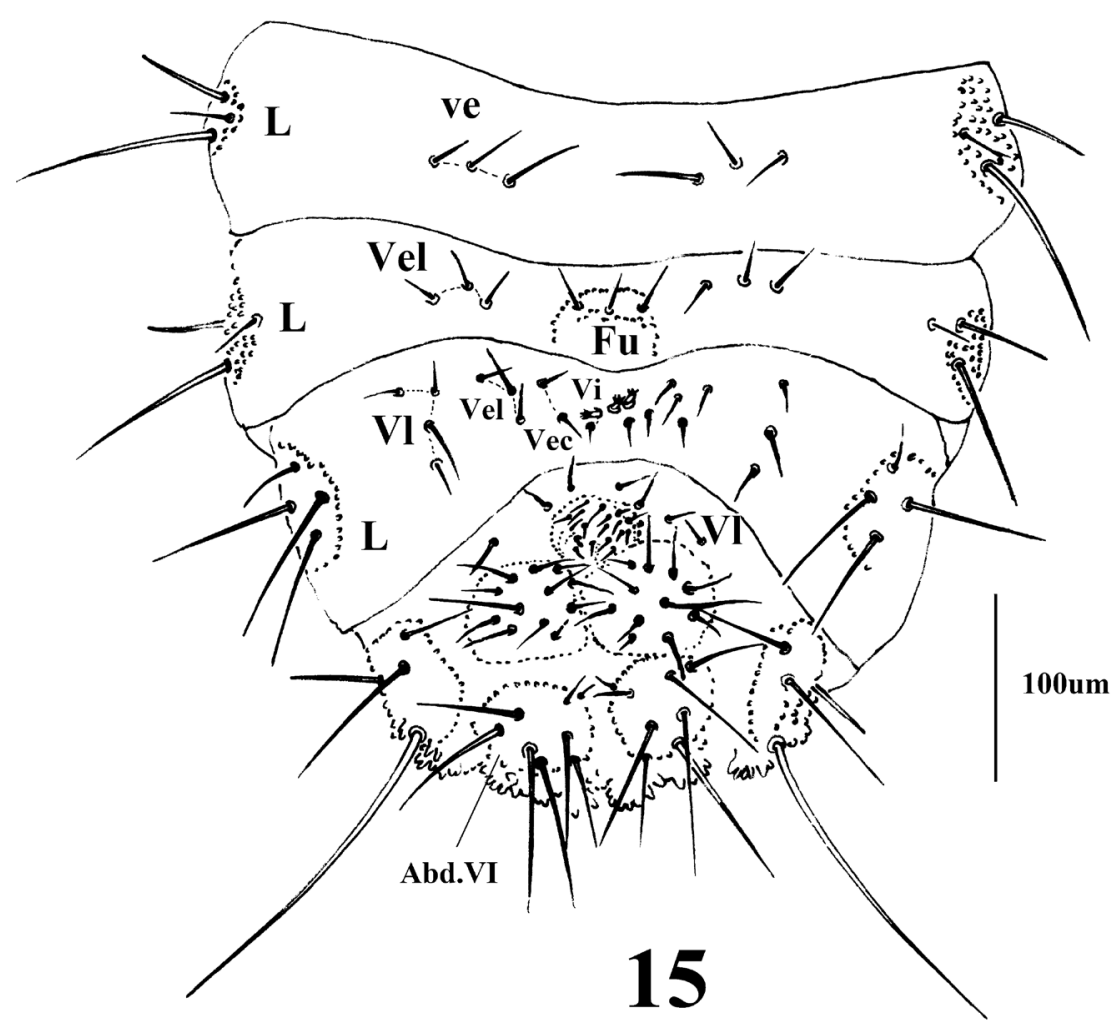

Figure I5. Vietnura caerulea Deharveng \& Bedos, 2000, ventral side of Abd. II-VI.

Vel with 3-4 chaetae. On Abd. IV, group Vei, Vec, Vel respectively with one, two, three chaetae, $\mathrm{Vl}$ with three or four chaetae. On Abd. V, group Vl with two chaetae, chaeta L' absent, Ag with two chaetae. Anal lobe with twelve chaetae and one mi.

Appendages. Unguis without tooth. Chaeta M on tibiotarsus present. Tibiotarsus of foreleg, midleg and hindleg, respectively with 19, 19, 18 chaetae. Chaetotaxy of ventral tube and furcular remnant as in Table 7.

Ecology and distribution. Among fallen leaves of bamboo and under broadleaved trees in the forest. The species is described from Vietnam. In China, it is only known from Maolan National Nature Reserve, Libo County (Fig. 16).

Remarks. Vietnura caerulea is easily distinguished among Chinese Neanurinae by its blue body color, six tubercles on the head, 2+2 pigmented eyes on tubercle Af+Oc, and reduced mandible and maxilla. Additionally, Ve chaetal group of Abd. IV has 3-5 shortened, thickened, and distally ciliated chaetae (male), claw is toothless, and hypotrichosis is developed on body tubercles. 


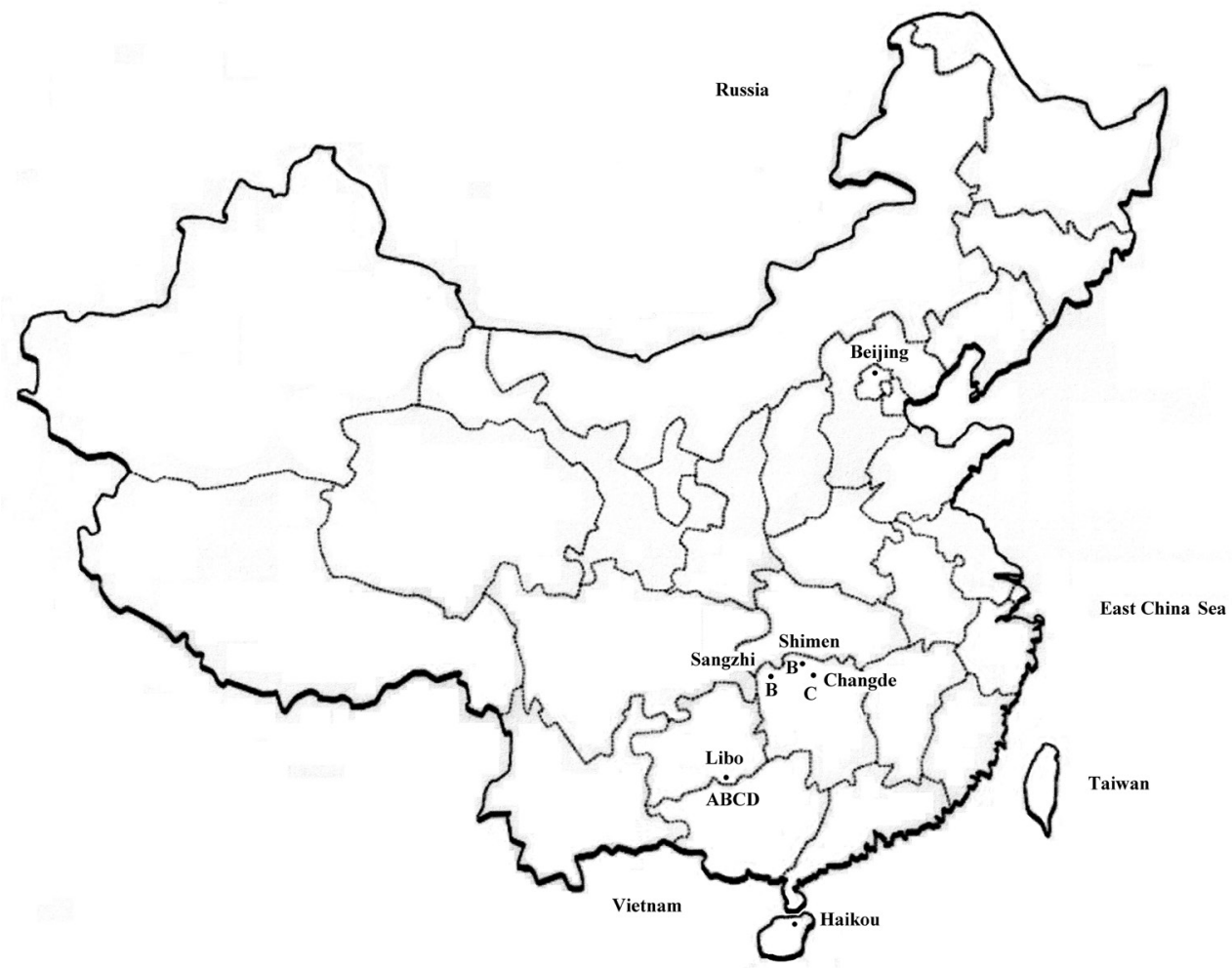

Figure 16. Map of China, with localities of Lobellina yinae sp. n. (A), Rambutanura hunanensis (B), Vitronura paraacuta $(\mathbf{C})$ and Vietnura caerulea (D).

\section{Tribe Paleonurini Cassagnau, 1989}

Genus Rambutanura Deharveng, 1988

\section{Rambutanura hunanensis Jiang \& Dong, 2018}

Rambutanura hunanensis Jiang \& Dong, 2018: 377-386, figs 1 -14 (China)

Material. One juvenile, body length $2.2 \mathrm{~mm}$, on slide; two specimens in alcohol, probably juvenile. Maolan National Nature Reserve, Libo County, Guizhou Province, China, $25^{\circ} 16.400^{\prime} \mathrm{N}, 107^{\circ} 53.864^{\prime} \mathrm{E}$, ca. $890 \mathrm{~m}$ above sea level. 19 July 2015 . Collected by Cheng-Wang Huang, Yan Liang, and Ai-Min Liu.

Diagnosis. The specimen from Libo County is characterized by its body without long digitate tubercles and tertiary granules, $2+2$ depigmented eyes, mandible with four teeth, maxilla styliform, head with eight tubercles (Cl, Af, 2 Oc, $2 \mathrm{Di}+\mathrm{De}, 2$ $\mathrm{Dl}+\mathrm{L}+\mathrm{So}$ ), claw with a big inner tooth, and ventral tube with 5-6 chaetae. These characters are similar to those of Rambutanura hunanensis Jiang \& Dong, 2018 from 
Hunan Province; however, the presence of only four chaetae on genital plate reveals the immaturity of the Maolan specimens.

Remarks. The distribution of $R$. hunanensis is given in Fig. 16. The species has been collected from other localities in China, such as Huping Mountain, Shimen County, Hunan Province (unpublished). It is probably widely distributed in central and southwest China.

\section{Genus Vitronura Yosii, 1969}

\section{Vitronura paraacuta Wang, Wang \& Jiang, 2016}

Vitronura paraacuta Wang, Wang \& Jiang, 2016: 183-196, figs 1-7 (China)

Material. Two females, submature, on slides, five specimens in alcohol, Maolan National Nature Reserve, Libo County, Guizhou Province, China, $25^{\circ} 16.400^{\prime} \mathrm{N}$, $107^{\circ} 53.864^{\prime} \mathrm{E}$, ca. $880 \mathrm{~m}$ above sea level. 19 July 2015. Collected by Cheng-Wang Huang, Yan Liang, and Ai-Min Liu.

Diagnosis. The characters of the specimens from Maolan are consistent with those of Vitronura paraacuta Wang, Wang \& Jiang, 2016: body tubercles well differentiated, head with 14 tubercles (only cephalic tubercle L fused to So), $2+2$ depigmented eyes, mandible with four teeth, maxilla styliform, tubercles Fr and Oc with three chaetae each, and claw with an inner tooth.

Remarks. The arrangement of the dorsal body tubercles and numbers of chaetae on dorsal tubercles of $V$. paraacuta are very similar to those of $V$. dentata Deharveng \& Weiner, 1984 from Korea. However, V. paraacuta can be differentiated from V. dentata by almost smooth body macrochaetae, four teeth on mandible, chaetae Di2, De2 on cephalic tubercle De and chaeta Oca on cephalic tubercle Oc being mesochaetae (vs serrated body macrochaetae, three teeth on mandible, chaetae Di2, De2 on cephalic tubercle De and chaeta Oca on cephalic tubercle Oc being microchaetae in $V$. dentata). The distribution of $V$. paraacuta is given in Fig. 16.

\section{Acknowledgements}

This study was supported by the National Natural Science Foundation of China (31772510, 31501849, 31471958), the Open Fund Project of Innovation Platform for University of Hunan Province (No. 14k065), the Natural Science Fund of Hunan Province (No. 2016JJ2091) and the Science Research Fund of Hunan University of Arts and Science (13ZD05). We thank two referees, Adrian Smolis (University of Wrocław, Poland) and Louis Deharveng (Muséum national d'Histoire naturelle, France), for their valuable suggestions and comments on the manuscript. 


\section{References}

Cassagnau P (1983) Un nouveau modèle phylogénétique chez les Collemboles Neanurinae. Nouvelle Revue d'Entomologie 13(1): 3-27.

Cassagnau P, Deharveng L (1984) Collemboles des Philippines. I - Les lobelliens multi colores des montagnes de Luzon. Travaux du Laboratoire d'Écobiologie des Arthropodes Edaphiques, Toulouse 5(1): 1-11.

Deharveng L (1983) Morphologie évolutive des Collemboles Neanurinae en particulier de la lignée Neanurinae. Travaux du Laboratoire d'Écobiologie des Arthropodes Edaphiques, Toulouse 4(2): 1-63.

Deharveng L, Bedos A (2000) Vietnura caerulea new genus, new species from Vietnam: First record of the Palaearctic tribe Neanurini in tropical Asia (Collembola: Neanuridae). Raffles Bulletin of Zoology 48(2): 209-214.

Deharveng L, Weiner W (1984) Collemboles de Corée du Nord III- Morulinae et Neanurinae. Travaux du Laboratoire d'Écobiologie des Arthropodes Edaphiques, Toulouse 4(4): 1-61.

Dong L, Yang YL, Zhao YY, Wang YJ, Jiang JG (2018) First record of the tropical genusRambutanura (Collembola: Neanuridae: Neanurinae) in Palearctic, with description of a new species from China. Zootaxa 4378(3): 377-386. https://doi.org/10.11646/zootaxa.4378.3.6

Jiang JG, Wang WB, Xia H (2018) Two new species ofLobellinifrom Tianmu Mountain, China (Collembola,Neanuridae). ZooKeys 726: 1-14. https://doi.org/10.3897/ zookes.726.11934

Lee BH (1980) Two Neanurid species of Collembola (Insecta) from Korea with polytene chromosomes in salivary glands. Korean Journal of Zoology 23(4): 251-262.

Ma ZC, Chen JX (2008) A new species of Lobellina (Collembola: Neanuridae) from China. Zootaxa 1733: 61-67.

Smolis A, Deharveng L (2006) Vitronura mascula, a new species of Neanurinae (Collembola: Neanuridae) from northern Vietnam, with a key to the species of the genus. Revue Suisse de Zoologie 113(2): 263-268. https://doi.org/10.5962/bhl.part.80349

Smolis A (2008) Redescription of four Polish Endonura Cassagnau, 1979 (Collembola, Neanuridae, Neanurinae), with a nomenclature of the ventral chaetae of antennae. Zootaxa 1858: 9-36.

Smolis A (2017) Contribution to the knowledge of Neanurinae of Vietnam with description of three new species (Collembola, Neanuridae). ZooKeys 688: 15-23. dhttps://doi. org/10.3897/zookeys.688.12307

Stach J (1965) On some Collembola of North Vietnam. Acta Zoologica Cracoviensia 10(4): 345-372.

Wang YR, Wang WB, Jiang JG (2016) New Neanurinae (Collembola: Neanuridae) from Central China. Zootaxa 4139(2): 183-196. http://doi.org/10.11646/zootaxa.4139.2.3

Yosii R (1954) Springschwänze des Ozé-Naturschutzgebietes. Scientific Researches of the Ozegahara Moor, 777-830.

Yosii R (1956) Höhlencollembolen Japans II. Jappanese Journal of Zoology 11(5): 610-627.

Yosii R (1969) Collembola-Arthropleona of the IBP-Station in the Shiga Heights, Central Japan, I. Bulletin of the National Science Museum 12(3): 531-556.

Yosii R (1976) On some Neanurid Collembola of Southeast Asia. Nature and life in southeast Asia 7: 257-299. 